\title{
Pregenual Anterior Cingulate Dysfunction Associated with Depression in OCD: An Integrated Multimodal fMRI/'H MRS Study
}

\author{
Reza Tadayonnejad', , Rangaprakash Deshpande', Olusola Ajilore'², Teena Moody', Francesca Morfini', \\ Ronald Ly ${ }^{1,3}$, Joseph O'Neill ${ }^{3}$ and Jamie D Feusner' \\ 'Division of Adult Psychiatry, UCLA Semel Institute for Neuroscience and Human Behavior, Los Angeles, CA, USA; ${ }^{2}$ Department of Psychiatry, \\ University of Illinois at Chicago, Chicago, IL, USA; ${ }^{3}$ Division of Child and Adolescent Psychiatry, UCLA Semel Institute for Neuroscience and Human \\ Behavior, Los Angeles, CA, USA
}

\begin{abstract}
Depression is a commonly occurring symptom in obsessive-compulsive disorder (OCD), and is associated with worse functional impairment, poorer quality of life, and poorer treatment response. Understanding the underlying neurochemical and connectivity-based brain mechanisms of this important symptom domain in OCD is necessary for development of novel, more globally effective treatments. To investigate biopsychological mechanisms of comorbid depression in OCD, we examined effective connectivity and neurochemical signatures in the pregenual anterior cingulate cortex (pACC), a structure known to be involved in both OCD and depression. Resting-state functional magnetic resonance imaging ( $\mathrm{fMRI}$ ) and ' $\mathrm{H}$ magnetic resonance spectroscopy (MRS) data were obtained from participants with $\operatorname{OCD}(n=49)$ and healthy individuals of equivalent age and sex $(n=25)$. Granger causality-based effective (directed) connectivity was used to define causal networks involving the right and left pACC. The interplay between $\mathrm{MRR}$ connectivity, 'H MRS and clinical data was explored by applying moderation and mediation analyses. We found that the causal influence of the right dorsal anterior midcingulate cortex (daMCC) on the right PACC was significantly lower in the OCD group and showed significant correlation with depressive symptom severity in the OCD group. Lower and moderate levels of glutamate (Glu) in the right pACC significantly moderated the interaction between right daMCC-pACC connectivity and depression severity. Our results suggest a biochemical-connectivity-psychological model of pACC dysfunction contributing to depression in OCD, particularly involving intracingulate connectivity and glutamate levels in the pACC. These findings have implications for potential molecular and network targets for treatment of this multi-faceted psychiatric condition. Neuropsychopharmacology (2018) 43, I |46-I 155; doi:I0.1038/npp.2017.249; published online 29 November 2017
\end{abstract}

\section{INTRODUCTION}

Comorbid depressive disorders, and co-occurring depressive symptoms, are relatively common in obsessive-compulsive disorder (OCD) and present additional layers of suffering and disability as well as posing added challenges for treatment. Epidemiological studies have shown lifetime prevalence of $40-70 \%$ and $13-24 \%$, respectively, for major depressive disorder and dysthymic disorder among patients with an OCD diagnosis (Murphy et al, 2013; Ruscio et al, 2010). Interestingly, the onset of OCD was found more often to precede the occurrence of depression (Ruscio et al, 2010) suggesting a causal model in which OCD for many is a strong predisposing factor for developing depression (Besiroglu et al, 2007). Several studies reported that

* Correspondence: Dr R Tadayonnejad, Room \# 27.432, UCLA Semel Institute for Neuroscience and Human Behavior, 760 Westwood Plaza, Los Angeles, CA 90024, USA, Tel: 310206 9632, Fax: 3102068387, E-mail: RTadayonNejad@mednet.ucla.edu

Received 25 February 2017; revised 18 September 2017; accepted 15 October 2017; accepted article preview online 20 October 2017 functional impairment and quality of life is worse in OCD patients with comorbid depression compared to nondepressed OCD patients (Stengler-Wenzke et al, 2007; Tukel et al, 2006). From a treatment perspective, comorbid depression is associated with significantly poorer response to psychological treatment and earlier drop-outs from psychological and pharmacological treatments in individuals with OCD (Abramowitz et al, 2000; Aderka et al, 2011; Keeley et al, 2008). In all, these findings underscore the importance of improving our understanding of depression in OCD and the necessity to develop novel and more effective treatment options for managing this difficult constellation of psychiatric symptoms.

Findings from biological research, particularly neuroimaging, in OCD and depression consistently point to a 'circuit dysfunction' as an underlying neural mechanism rather than a specific regional abnormality. Aberrant function in corticostriatal-thalamo-cortical (CSTC) neurocircuitry is strongly associated with OCD pathology (Ahmari and Dougherty, 2015; Saxena and Rauch, 2000), while the evidence in depression implicates dysfunction in fronto-limbic networks 
(Tadayonnejad and Ajilore, 2014). Pregenual anterior cingulate cortex (pACC) is an important midline structure involved in emotional processing and affect regulation and plays an essential role in depression neurocircuitry (Pizzagalli, 2011). It also has strong anatomical/functional connections with regions involved in CSTC circuits (Beckmann et al, 2009; Johansen-Berg et al, 2008). Interestingly, functional abnormalities of the pACC have been reported in both OCD (Breiter et al, 1996; Mataix-Cols et al, 2004) and depression (Kennedy et al, 2001). Neurochemical abnormalities of the pACC have also been found in both OCD (O'Neill et al, 2016) and depression (Ernst et al, 2016), with more consistent findings in the latter. Among the major neurochemicals showing abnormalities in both OCD and depression is glutamate (Glu), the main excitatory neurotransmitter in the brain, which has motivated the formulation of glutamate hypotheses of depression (Sanacora et al, 2012) and OCD (Rosenberg and Keshavan, 1998). For review see Pittenger et al (2011). It should be noted that Glu findings in OCD, particularly in the pACC, are not consistent; some studies reported no effects of OCD on glutamatergic compounds in pACC (Simpson et al, 2012; Yucel et al, 2008). Differences in results between studies may relate to methodological factors, for example, variation in brain region sampled and patient medication. We also recently found abnormally high levels of glutamate+glutamine (Glx) in the pACC in OCD (O'Neill et al, 2016). Based on this reported biochemical and functional pathology in both OCD and depression, and its connectivity profile, the pACC is an important target for exploring brain mechanisms of comorbid depression in OCD, and Glu may be a key regional neurochemical to investigate.

Recent advances in neuroimaging and analysis methods have provided opportunities for deeper exploration of brain neurochemistry, structure, function, and their interrelationships in healthy brains as well in neuropsychiatric conditions. In OCD, although functional magnetic resonance imaging (fMRI) studies have shown mostly consistent findings converging on CSTC malfunction in OCD (Ahmari and Dougherty, 2015), biochemical research, primarily using magnetic resonance spectroscopy (MRS), has resulted in relatively inconsistent findings. This may be due to methodological differences or to the heterogeneous nature of OCD (Brennan et al, 2013), not only in regards to core OCD symptoms but also comorbidities and commonly co-occurring symptoms, such as anxiety and depression. Another possible source of variable results is that neurometabolites detected by MRS may play a mediatory or modulatory, rather than a direct, role in OCD. In particular, the original glutamate hypothesis of OCD referred to mediation of the function and metabolism of anterior cingulate-striatal circuits by Glu (Rosenberg and Keshavan, 1998). Such mediatory or modulatory relationships might be identified using multimodal imaging, but this has been sparsely applied to date in OCD (Brennan et al, 2015; Yucel et al, 2007). Specifically, in OCD research there has been the lack of integration between biochemical (MRS) and functional (fMRI) investigations, which makes it difficult to achieve a multidimensional biological model of brain dysfunction in OCD, with or without common comorbidities. One approach to address those methodological issues is the use of integrated multimodal imaging approaches, which have been sparsely applied to OCD neuroimaging research (Brennan et al, 2015; Yucel et al, 2007).

In the current study, we applied a multimodal imaging approach, combining resting-state fMRI and ${ }^{1} \mathrm{H}$ MRS data acquisition to investigate brain biochemical-network connectivity mechanisms of comorbid depression in OCD. We focused on the pACC because of its unique involvement in both OCD and depression pathology. Furthermore, we applied mediation and moderation analysis methods to explore the dynamics of interaction between neurochemical, network and clinical symptomology factors implicated in comorbid depressive symptoms in OCD rather than relying on a simple correlation approach. Considering the aforementioned accumulating evidence for the pACC involvement in both OCD and depression pathology as well as the widespread anatomical connections of the pACC with CSTC nodes, we hypothesized that: (1) pACC network causal interactions, estimated by Granger Causality-based effective connectivity analysis, would differ between individuals with OCD and healthy controls; and (2) the degree of pACC effective connectivity would be significantly associated with clinical OCD and depression symptoms in OCD individuals. In the context of reports of Glu involvement in the pathophysiology of both OCD and depression, and in accordance with the glutamate hypotheses of OCD and depression, we proposed an exploratory hypothesis in which we predicted that (3) the level of Glu in the pACC would either mediate or moderate the association between fMRI connectivity and clinical symptoms in individuals with OCD.

\section{MATERIALS AND METHODS}

\section{Participants}

Forty-nine individuals with DSM-IV OCD and 25 healthy control participants (HC) were recruited from UCLA and other clinics or by advertisement. Informed consent was provided for all participants, and the UCLA Institutional Review Board approved the study. A board certified psychiatrist (JDF) with clinical experience with OCD established the diagnoses after performing comprehensive evaluations. Additional details of the clinical assessments of study participants are provided in Supplementary Information.

\section{fMRI Data Acquisition and Preprocessing}

Whole-brain blood oxygenation level-dependent (BOLD) fMRI was acquired using a Siemens Trio 3T scanner (Siemens Medical Solutions USA Inc., Malvern, Pennsylvania) with a 12-channel phased-array head coil during a 7min resting-state, eyes closed scan. Additional details regarding fMRI data acquisition and preprocessing and head motion assessments are provided in Supplementary Information.

\section{fMRI Deconvolution}

The 3D+time fMRI data obtained after pre-processing were deconvolved across time at every voxel to obtain estimates of the latent neural time series ( $\mathrm{Wu}$ et al, 2013), which were then used in further analyses. Deconvolution minimizes the 
confound of non-neural hemodynamic response function variability inherent in the BOLD fMRI signal (Handwerker et al, 2004), and is an approach that several recent fMRI studies have employed (Boly et al, 2015). Please refer to Supplementary Information for a detailed account.

\section{${ }^{1} \mathrm{H}$ MRS Acquisition and Post-Processing}

In the same session as $\mathrm{PMRI}$, localized water-suppressed MRS (PRESS; TR/TE $=2000 / 30 \mathrm{~ms}$, 96 NEX, voxels $\sim 15 \times 15 \times 15 \mathrm{~mm}^{3}$ ) was obtained from right and left pACC, followed by identical non-water-suppressed MRS ( $8 \mathrm{NEX)} \mathrm{at}$ each site. The principal metabolite endpoint was MRS glutamate (Glu), not glutamate+glutamine (Glx). Wholebrain structural MRI (MPRAGE, $1 \times 1 \times 1 \mathrm{~mm}^{3}$ ) was also acquired in order to prescribe MRS and for post-processing of MRS and fMRI. MPRAGE was segmented into gray matter, white matter, and CSF and Glu levels were adjusted for voxel tissue composition. Please refer to Supplementary Information for more details.

\section{Effective Connectivity Seed Selection}

The right and left pACC regions of interest were derived by transforming each participant's MRS voxel's centroid to the standard MNI space and then averaging them across all participants to obtain mean centroids. The seeds were then defined as $7.5 \mathrm{~mm}$-radius spheres around these mean centroids. Please see Supplementary Information for further details.

\section{Granger Causality (GC)-Based Effective (Directed) Connectivity Analysis}

In this step of the analysis we first established the effective connectivity causal network with the pACC across all participants. GC-based bivariate seed-to-whole-brain and whole-brain-to-seed effective connectivity (EC) analyses were performed on deconvolved resting-state fMRI data to examine the causal interaction between the pACC and the rest of brain, as described in our previous published studies (Tadayonnejad et al, 2016a,b). This generated four sets of causal networks: right pACC to whole brain, left pACC to whole brain, whole brain to right pACC, and whole brain to left pACC. Additional details are provided in Supplementary Information.

\section{Statistical Analysis}

We performed two statistical analysis steps to test and investigate our main a priori hypothesis of the study that abnormal effective connectivity between pACC and other CSTC brain regions is associated with depression symptoms in OCD, following from the evidence reviewed above. In step 1 , which was more data-driven, by taking left and right pACC as seeds, we used GC analysis across the entire (OCD $+\mathrm{HC}$ ) sample to calculate the EC from each seed to every other region in the whole-brain network and vice versa. We used FDR to threshold for statistically significant connections at the conservative value of $q=10^{-5}\left(P=5.8 \times 10^{-8}\right)$. This yielded four networks of significant connections: regions with causal influence on right pACC, regions with causal influence on left pACC, regions that receive causal influence from right $\mathrm{pACC}$, and regions that receive causal influence from left pACC. Step 2 then examined betweengroup differences in EC within each of these networks. Invoking the CSTC-side of our a priori hypothesis, we only considered connections between pACC and other CSTC sites. Moreover, between-group comparisons were Bonferroni-corrected for the number of pACC-CSTC connections within each network to determine the final effects of group on EC. Associations within the OCD group between EC for each pACC-CSTC connection and scores on clinical symptom-domain instruments underwent similar Bonferroni correction. All statistical analyses were performed with SPSS (IBM SPSS Statistics for Windows, Version 24.0. Armonk, NY). Additional details are provided in Supplementary Information.

\section{Mediation and Moderation Analyses}

Motivated by the glutamatergic hypotheses of depression and OCD, as explained above, for each pACC-CSTC node connection showing a significant relation between connectivity and the Montgomery-Åsberg Depression Rating Scale (MADRS) score within the OCD sample, we sought to determine whether pACC MRS Glu level was functioning as a variable through which connectivity influences MADRS score (mediation analysis) or whether pACC Glu level determined the boundary condition for connectivityMADRS interaction (moderation analysis). Mediation and moderation analyses were performed by using Hayes's method of ordinary least squares regression-based path analysis, implemented in PROCESS macro (run in SPSS), which also includes bootstrap-based confidence interval calculations (Hayes, 2013). Please see Supplementary Information for more details.

\section{RESULTS}

\section{Demographics and Clinical Characteristics}

Demographic and clinical information for both healthy and OCD participants are summarized in Table 1 . There were no

Table I Demographic and Clinical Characteristics of Patients and Control Subjects

\begin{tabular}{|c|c|c|c|}
\hline & HC $(n=25)$ & OCD $(n=49)$ & $P$-value \\
\hline & Average (SD) & Average (SD) & \\
\hline Age & $30.7 \pm 11.76$ & $33.3 \pm 11.03$ & $0.36^{\mathrm{a}}$ \\
\hline Sex (Male/Female) & $|4 /| \mid$ & $26 / 23$ & $0.058^{b}$ \\
\hline Education in years & $15.4 \pm 2.23$ & $15.5 \pm 2.32$ & $0.76^{\mathrm{a}}$ \\
\hline Y-BOCS & & $25.0 \pm 4.43$ & \\
\hline MADRS & $1.0 \pm 1.22$ & $15.2 \pm 8.54$ & $<0.001^{a}$ \\
\hline HAMA & $1.3 \pm 1.21$ & $12.2 \pm 5.79$ & $<0.001^{a}$ \\
\hline
\end{tabular}

Abbreviations: HAMA, Hamilton Anxiety Scale; HC, healthy control; MADRS,

Montgomery-Asberg Depression Scale; OCD, obsessive-compulsive disorder;

Y-BOCS, Yale-Brown Obsessive-Compulsive Scale.

a The $P$-value was obtained by sample $t$-test.

${ }^{\mathrm{b}}$ The $P$-value was obtained by chi-square test. 
Table 2 Brain Areas with Significant Causal Network Interaction with the Right and Left pACC across the Whole Sample of ObsessiveCompulsive Disorder and Healthy Control Participants $\left(n=74\right.$; FDR corrected, $q=10^{-5}$ )

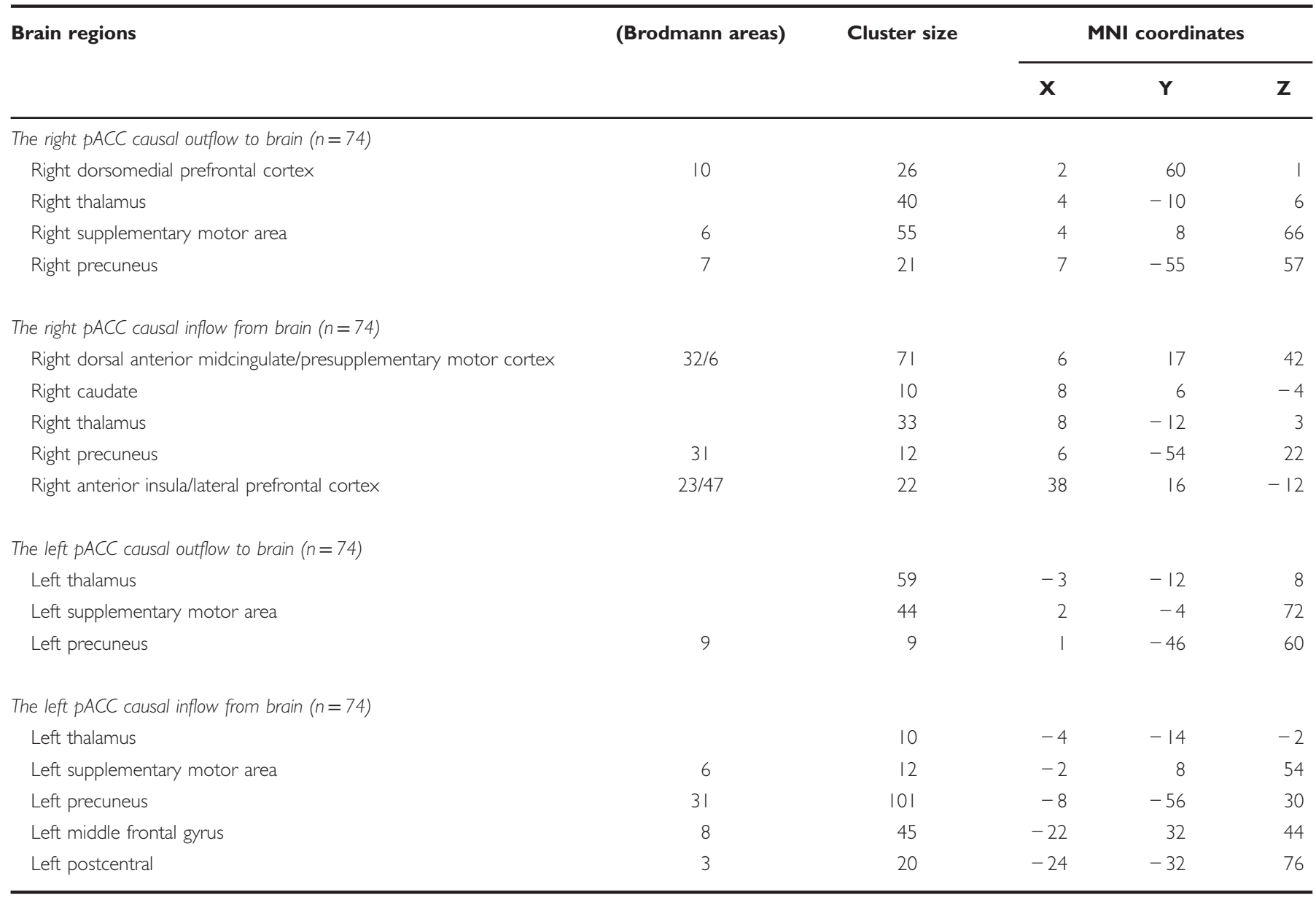

Abbreviations: BA, Brodmann area; pACC, pregenual anterior cingulate cortex; MNI, Montreal Neurological Institute.

significant differences between the two groups in age, gender or years of education. As expected, we found that OCD participants scored significantly higher than healthy participants on depression and anxiety symptom severity scales as measured by MADRS and the Hamilton Anxiety Scale (HAMA), respectively.

\section{OCD and Healthy Participant pACC Effective Connectivity}

Across the whole sample (OCD and healthy participants), both the right and left pACC seeds demonstrated causal interactions with different, primarily midline brain regions (Table 2). The pACC-to-whole-brain EC analyses showed that the right pACC has Granger causal influence on the right dorsomedial prefrontal cortex, thalamus, supplementary motor area and precuneus and the left pACC was found to have Granger causal influence on the left thalamus, supplementary motor area and precuneus $(n=74$; FDR corrected, $q=10^{-5}$ ). Whole-brain-to-pACC EC analysis revealed that the right $\mathrm{pACC}$ receives Granger causal influence from the right dorsal anterior midcingulate area (daMCC), caudate, thalamus, precuneus and anterior insula/ lateral orbitofrontal cortex compared to the left pACC that receives Granger causal influence from the left thalamus, supplementary motor area, precuneus, middle frontal gyrus and postcentral gyrus ( $n=74$; FDR corrected, $\left.q=10^{-5}\right)$.

Group comparison analysis between OCD $(n=49)$ and HC ( $n=25)$ groups showed that Granger causal influence of the right daMCC on pACC (right daMCC-to-pACC connectivity) is $42.9 \%$ lower in OCD participants compared to control group (HC: $0.07 \pm 0.008$; OCD: $0.04 \pm 0.006$; Bonferroni corrected, $P=0.013$ ) (Figure 1).

\section{Association between pACC Effective Connectivity and Clinical Symptoms}

Next, we assessed whether values of the right daMCC-pACC connectivity are related to clinical symptoms in the OCD group. We found a significant correlation between the value of the right daMCC-pACC connectivity and depression symptom severity in terms of MADRS score $(r=-0.38$, Bonferroni corrected, $P=0.008$; Figure 2). Considering the significant and relatively strong correlation between depression and OCD and anxiety symptom severity (MADRS vs Y-BOCS; $R=0.39, P=0.007$; MADRS vs HAMA; $R=0.84$, $P<0.001$ ), we expected that the right daMCC connectivity would also show correlation with OCD and anxiety symptom severity. However, right daMCC-pACC connectivity was correlated exclusively with depression but not OCD or 
anxiety symptoms (Figure $2 \mathrm{~b}$ and $\mathrm{c}$ ). We also performed partial correlation analysis between the right daMCC-pACC connectivity and depression symptom severity (MADRS scores) while controlling for anxiety, OCD, and both anxiety and OCD symptom severity. The results of partial correlation analyses were very similar to the bivariate correlation ( $r=-0.38, P=0.008$ when controlling for Y-BOCS score; $r=-0.38, P=0.009$ when controlling for HAMA score and $r=-0.39, P=0.008$ when controlling for both Y-BOCS and HAMA scores). All in all, these findings suggest the specificity of right daMCC-pACC connectivity as associated with depression symptoms more so than OCD or anxiety symptoms.

\section{Glutamate Mediation/Moderation of the Relationship between daMCC-pACC Connectivity and Depressive Symptom Severity}

Mediation and moderation analyses were performed to explore what role glutamate (Glu) levels in the right pACC region (as $M$ : mediator or moderator) plays in the modeled

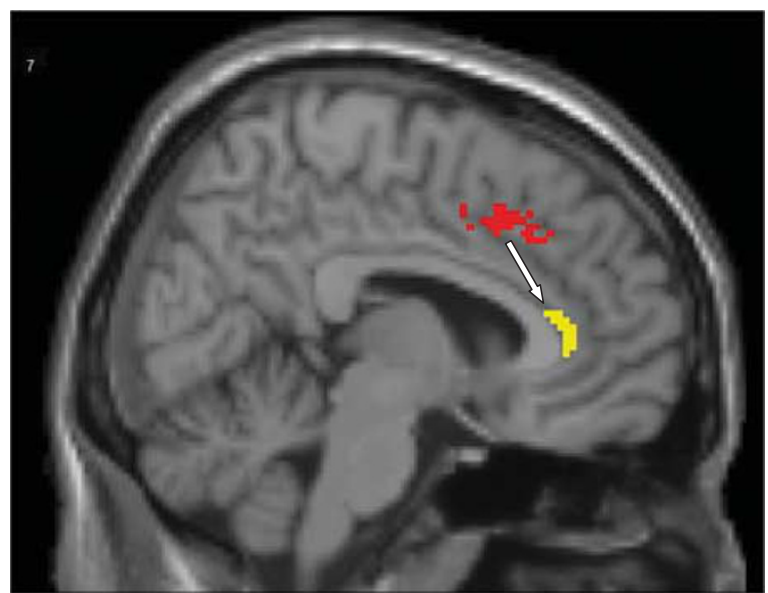

Figure I Sagittal view ( $\mathrm{TI}$ structural MRI) of the right dorsal anterior midcingulate cortex (daMCC) cluster (in red) in which there was significantly lower excitatory causal influence on the right pregenual anterior cingulate ( $\mathrm{PACC}$ ) seed (in yellow) in OCD participants compared to the healthy control group. MRI, magnetic resonance imaging; OCD, obsessivecompulsive disorder. interaction between the right daMCC-pACC connectivity (as $X$ : independent variable) and depression symptom severity (as $Y$ : dependent variable or outcome) in subjects with OCD. In an estimation model of daMCC-pACC connectivity effect on depression symptom severity, no significant mediatory role was found for the right pACC Glu level. Moderation analysis, however, showed that the right daMCC-pACC connectivity $(X)$ exerts a significant effect on depression symptom severity (Y) when lower or moderate levels of Glu exist (lower Glu level: $X$ Effect on $Y=-88.0, P=0.03$, lower levels for confidence interval (LLCI) $=-168.5$, upper levels for confidence interval (ULCI) $=-7.4$; moderate Glu level: $X$ Effect on $Y=-64.9, P=0.03, \quad \mathrm{LLCI}=-121.5, \mathrm{ULCI}=-8.2)$. In other words, significant effect of the right daMCC-pACC connectivity on depression is conditional on the lower and moderate Glu levels on the right pACC seed. Figure $3 \mathrm{a}$ is a visual demonstration of moderation analysis structure and results in which two-variable $(X, Y)$ linear regression was calculated separately for three different levels of Glu (lower, moderate and higher) (Figure $3 \mathrm{~b}$ ) showing how connectivity exerts the strongest effect on depression symptom severity scores for lower Glu levels $(R=0.61, P=0.02)$. Using the Johnson-Neyman method (Bauer and Curran, 2005), we also determined that Glu values between 28.5 and 38.8 IU represents 'the region' or range of Glu levels in which the right daMCC-pACC connectivity can significantly affect (interact with) depression symptom severity. For further validation of the moderation analysis results, we applied the moderation analysis on two (randomly) separated groups within the OCD sample with sample sizes of $n=20$ and $n=23$ (please see Supplementary Information for more details). Although this split-sample moderation analysis did not show any significant results in the two subgroups (likely due to low power), however, within each subgroup, the direction of $X$ effect on $Y$ (in other words, the correlation between connectivity and depressive symptoms) was always negative, similar to the findings of moderation analysis across all OCD participants, supporting a consistent direction of the findings.

\section{DISCUSSION}

This study investigated neurochemical and network mechanisms of the role of pACC in comorbid depressive symptoms in OCD, using a multimodal imaging approach that
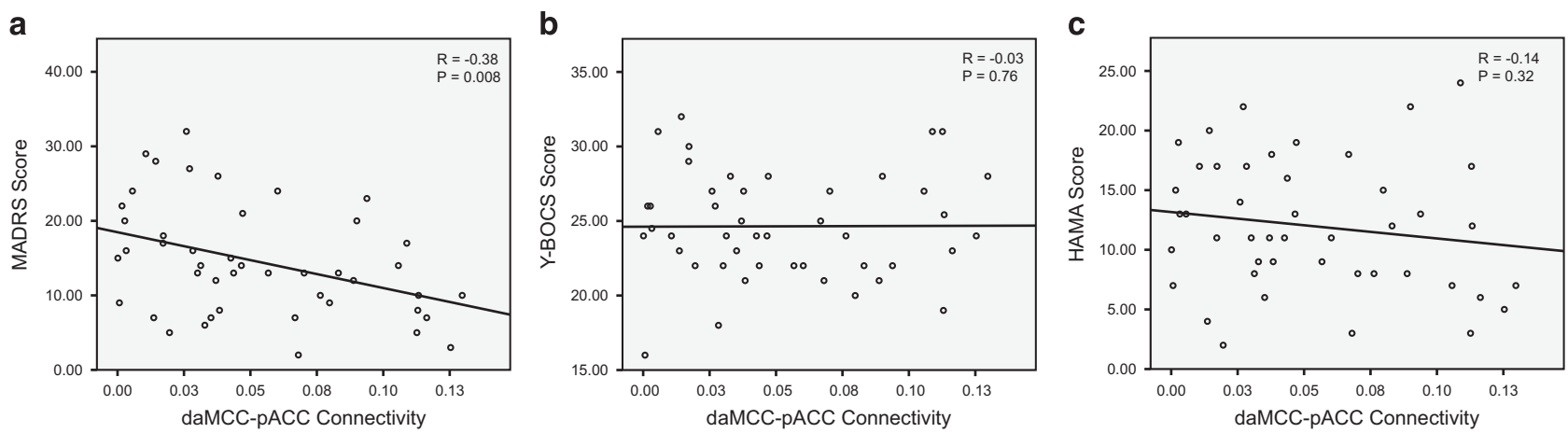

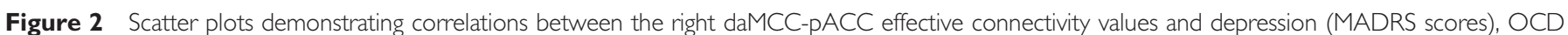

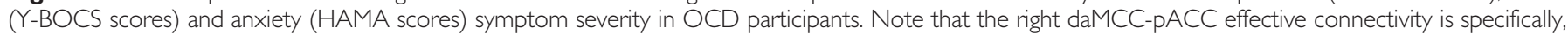

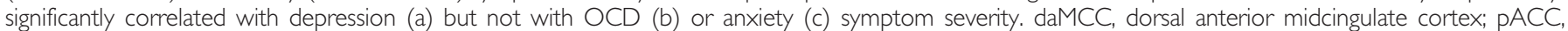

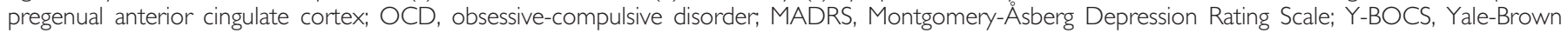
Obsessive-Compulsive Scale; HAMA, Hamilton Anxiety Scale. 

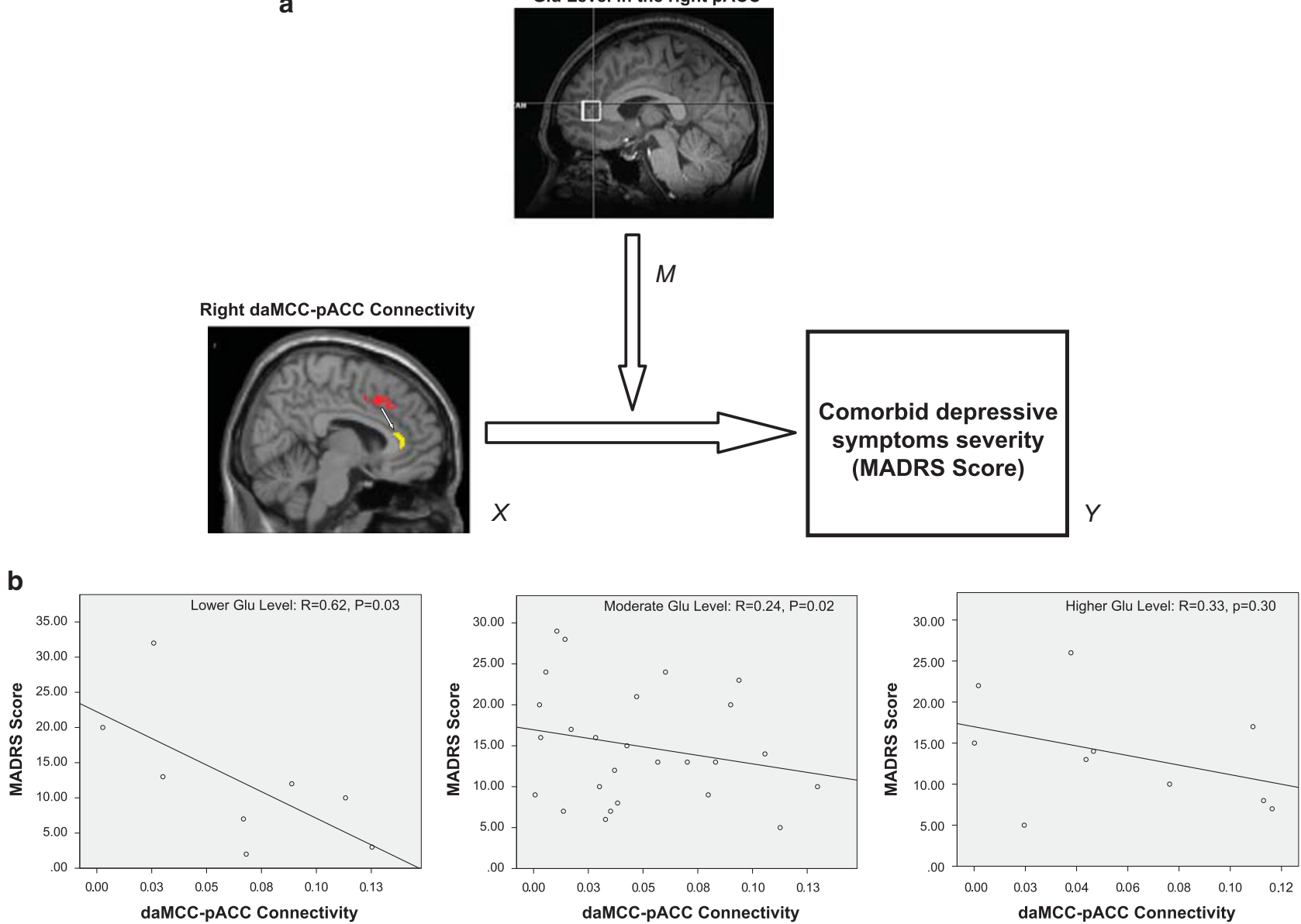

Figure 3 (a) Schematic demonstration of the moderation analysis with depressive symptom severity. MADRS is the output variable (Y), the right daMCCPACC connectivity is the independent variable (Y), and Glutamate (Glu) level in the right pACC is the moderator (M) between $X$ and $Y$. (b) Scatterplots illustrate the association between $X$ and $Y$ among OCD participants with relatively lower $(n=8$, Left), moderate $(n=25$, Middle) and higher $(n=10$, Right) levels of Glu in the right pACC seed. MADRS, Montgomery-Åsberg Depression Rating Scale; daMCC, dorsal anterior midcingulate cortex; pACC, pregenual anterior cingulate cortex; OCD, obsessive-compulsive disorder.

combined resting state fMRI effective connectivity and ${ }^{1} \mathrm{H}$ MRS. We found a significant lower casual influence of the right daMCC on pACC (daMCC-to-pACC effective connectivity) in OCD individuals compared to healthy controls. In the OCD group, a significant correlation was found between daMCC-to-pACC connectivity and exclusively depression symptom severity but not OCD or anxiety symptom severity. Furthermore, our moderation path analysis supports an estimating model in which right daMCC-to-pACC connectivity exerts a significant effect on depression symptom severity for lower or moderate Glu levels in the right pACC.

Before discussing our daMCC-pACC connectivity findings, it should be noted that 'causal' effective connectivity in our study refers to an estimation of excitatory (stimulatory) or inhibitory (dampening) influence of brain regions on each other estimated by the Granger Causality analysis method (please see Supplementary Information for more details of the Granger Causality method). Furthermore, we also need to establish a context by recalling briefly the functional brain networks in which these two structures are embedded. The daMCC, in conjunction with the dorsolateral prefrontal cortex and the superior parietal cortex, form the cingulofrontal-parietal cognitive/attention network (Glasser et al,
2016; Power et al, 2011; Yeo et al, 2011). Located more anteriorly, the pACC (also called the rostral ACC) combines with the ventromedial prefrontal cortex (vmPFC), posterior cingulate/precuneus, lateral parietal cortex, and dorsomedial prefrontal cortex (dmPFC) to form the default-mode network (DMN) (Glasser et al, 2016; Power et al, 2011; Yeo et al, 2011), an intrinsically organized functional network with self-referential functions like introspective processing, remembering the past or future planning (Buckner et al, 2008). The activity of the cognitive/attention network shows temporal anticorrelation or uncoupling with DMN activity in both the resting-state and during task conditions (Fox et al, 2005) which can be disrupted in aging or neuropsychiatric conditions (Franzmeier et al, 2017; Habeck et al, 2012). Furthermore, the pACC shows increased activity during emotionally laden tasks with limbic/paralimbic regions belonging to an 'affective network' that includes the amygdala, nucleus accumbens, and orbitofrontal cortex (Bush et al, 2000). The dynamics of the daMCCpACC interaction in the context of the cognitive/attention and affective networks are 'reciprocally inhibitory'; cognitive-laden tasks cause deactivation of the pACC whereas emotionally related tasks induce deactivation of the daMCC (Bush and Shin, 2006). A further point is the 
prominent role of daMCC in the circuit pathology of OCD. Structural, functional, and connectivity imaging studies frequently identify daMCC (often called 'dorsal ACC') as a key node in an OCD CSTC network (Fitzgerald et al, 2010; Mataix-Cols et al, 2004; Saxena and Rauch, 2000). It should be noted that the daMCC has also been the target of invasive psychosurgery approaches such as cingulotomy in refractory OCD cases (Baer et al, 1995). Interestingly, and in line with our findings, cingulotomy often results in alleviation of comorbid depressive symptoms beside its main effect on primary OCD symptom reduction (Brown et al, 2016; Dougherty et al, 2002). Connectivity studies in particular reveal hyperconnectivity of daMCC within this CSTC circuit in OCD (Beucke et al, 2012; Fitzgerald et al, 2010).

In the above context and based on our primary finding of below-normal daMCC-to-pACC effective connectivity in OCD, we proposed a network-based model in which OCDrelated regional (structural/functional) and network connectivity dysfunction of daMCC within CSTC circuit lead to an impairment in the regulatory effect of the daMCC on pACC and subsequent precipitating dysfunction of the pACC within the encompassing DMN. In other words, hyperactivity of the daMCC within the CSTC circuit in OCD comes at a cost of abnormal reduction in the regulatory causal action of daMCC on the pACC, which, in turn, disrupts function of the pACC and of the DMN to which it belongs. In line with this model are studies of comorbid depression in OCD that reveal self-focused, cognitively distorted symptomology, such as 'greater tendency to misinterpret the significance of intrusive thoughts' (Abramowitz et al, 2007) or 'autogenous obsessions' with strong association to self-related shame and guilt (Lee et al, 2005). This profile might be expected from DMN dysfunction in OCD patients with more significant depression compared to those without depression.

This picture is consistent with our second major finding, that daMCC-pACC connectivity correlated exclusively with depressive, but not with OCD (as we hypothesized) or anxiety symptom severity, despite the commonly observed strong correlation of these three symptom-domains among themselves (Murphy et al, 2013). We suggest that the selective association between daMCC-pACC connectivity and depression symptoms might be related to our putative pACC-focused connectivity model. The evidence of pACC involvement in depression is somewhat more established than pACC involvement in OCD or anxiety networks, as reviewed in the Introduction. Therefore, it is plausible to expect that pACC network abnormalities, particularly the dysfunction of causal influence toward pACC found in our study, might perturb the depression circuit more strongly, and possibly even exclusively, compared with OCD or anxiety circuits. The resultant psychological manifestation would be depressive symptoms rather than OCD or anxiety symptoms. This is consistent with the (Middleton, 2009) cingulocentric model of OCD, an update of the (Alexander et al, 1986) model of parallel cortico-basal gangliar circuits. Middleton argues for an emotion-anxiety depression circuit that includes the pACC fostering depressive symptoms that is anatomically separate from the extra-pACC ideationobsessional thoughts and action-compulsive behaviors circuits responsible for obsessive-compulsive symptoms. The apparent association of pACC inhibition with lower depressive, but not lower obsessive-compulsive, symptoms seen here may be a reflection of this anatomical separation. It is more challenging to explain the absence of a correlation with anxiety symptoms. Middleton notes, however, that the neurophysiological effects of anxiety undergirding his model are localized to the caudal portions of the pACC, that is, those close to the daMCC, and within the daMCC. Moreover, in an analysis of resting-state fMRI data from healthy subjects varying widely in level of anxiety and controlling for depressed affect (Bijsterbosch et al, 2014) located independent effects of anxiety in numerous brain regions outside the pACC. Thus, anxiety symptoms may be less affected by the putative daMCC inhibition of pACC construed in the present findings. Granger EC may, furthermore, offer a means of anatomically disentangling clinically highly intercorrelated symptoms. That being said, to the best of our knowledge, no study has examined the whole brain restingstate effective connectivity of pACC, or daMCC-pACC effective connectivity during task performance, in noncomorbid OCD or anxiety disorders. Therefore, our results cannot rule out the possibility of daMCC-pACC connectivity involvement in non-comorbid OCD or anxiety disorders symptom generation. This is an important subject that needs to be explored in future works.

The third major finding was that higher (right) daMCCpACC connectivity was significantly associated with less severe depressive symptoms for lower and moderate, but not for higher, levels of pACC Glu. In interpreting the foregoing finding it is important to note that MRS measures wholetissue Glu, lacking the required spatial resolution for distinguishing between intra- and extracellular Glu, synaptic and extrasynaptic Glu, or neuronal and glial Glu. MRS is also limited in its ability to detect the exact cell-specific (pyramidal $v s$ interneuron) or subneuronal compartment (cell body, vs proximal or distal axon, $v s$ dendrite) source of Glu signal. These are all inherent limitations of the method making any microcircuitry level interpretation of MRS findings speculative in nature. Considering these methodological limitations, we suggest one speculative model for interpretation of our MRS findings (although it is by no means the only possible model). In this model, we assume a specific pACC microcircuitry architecture in which glutamatergic inputs from daMCC synapse upon GABAergic interneurons to generate local inhibition in pACC (daMCC excitatory neurons $\rightarrow$ pACC inhibitory interneuron $\rightarrow$ pACC excitatory neurons). Indirect support for this microcircuitry pattern comes from (a) cytology and receptor architecture studies showing the highest GABA receptor density (compared to other receptors) and a ratio lower than 1 of AMPA/GABA and NMDA/GABA in the pACC (Vogt et al, 2013), and (b) functional evidence of anticorrelation or reciprocal inhibition of daMCC and pACC (Bush and Shin, 2006). Following from this speculative model, inadequate Glu levels in the pACC might cause insufficient stimulation of AMPA receptors on pACC interneuron, with the subsequent effect of disinhibition of pACC excitatory pyramidal neurons that receive inhibition from surrounding interneurons. Insufficient Glu in pACC might also impact the normal activation of NMDA receptors, disturbing neuroplastic changes at daMCC-pACC interneuron synapses. Both of these mechanisms could contribute to a reduction of the regulatory effect of daMCC on pACC, 
subsequently leading to abnormal pACC interactions within the DMN and the affective network, resulting in depressive symptoms. Of course, this speculative model is based on a limited assumption that the main source of measured Glu signal comes from the daMCC afferents to interneurons and does not take into account other plausible sources including other cortical as well as thalamic afferent glutamatergic projections to pACC, or the Glu pool in the pACC pyramidal neurons cell bodies.

Our findings may have several future potential clinical translational implications. The correlation and moderation analyses results suggest two targets for management of comorbid depression in OCD: (a) connectivity of the right daMCC with pACC, and (b) Glu levels in the pACC. Neuromodulation techniques like tDCS with a specific montage that can target the daMCC and the daMCC-pACC white matter connecting tract could be used to enhance the right daMCC drive and its causal effect (effective connectivity) on the pACC. In a recent study, it was shown that methylphenidate can strengthen corticocortical connections including the one between dACC (daMCC) and rostral ACC (pACC) in subjects with cocaine addiction (Konova et al, 2013). A similar mechanism might also work in depression in OCD to increase the hypoconnectivity of the daMCCpACC, but would require testing. Another approach could be to enhance glutamergic functions in the pACC by targeting AMPA and NMDA receptors using AMPA receptor potentiator agents (Black, 2005) or NMDA partial agonists like rapastinel (GLYX-13), a novel agent under development for treatment of refractory depression (Moskal et al, 2016). Recently, and in line with our predicative model, administration of rapastinel was found to significantly reduce comorbid depression symptom severity (as well as main OCD symptoms) in subjects with OCD in a preliminary study (Rodriguez et al, 2016).

The current study has several methodological limitations. The cross-sectional design of the study does not permit differentiation between state-dependent and trait-dependent findings. Some OCD participants were medicated at the time of scanning, which might confound the fMRI and MRS results (Tadayonnejad et al, 2016a). The sluggish nature of fMRI BOLD signal and the variability of hemodynamic delay between brain areas might result in spurious Granger causality findings. We addressed this limitation by applying deconvolution on the raw resting-state fMRI data before performing Granger causality analysis (Wu et al, 2013), although it still provides an estimate (albeit improved) of the neural signal.

In summary, this study used a multimodal resting-state $\mathrm{fMRI} /{ }^{1} \mathrm{H}$ MRS approach to explore biochemical-networkfunctional role of the pACC in depressive symptoms in OCD. We found OCD is associated with a reduction in causal influence of the right daMCC on pACC showing specific correlation with depressive symptoms severity. Moreover, lower and moderate levels of Glu in the right pACC significantly moderate the interaction between daMCC-pACC connectivity and depressive symptoms. Based on these findings, we suggest novel network and biochemical targets for treatment of this relatively common and often poorly tractable condition of depression symptomatology in OCD, which warrant testing in future studies.

\section{FUNDING AND DISCLOSURE}

This study was supported by NIMH grants R01 MH085900 (to Drs Feusner and O’Neill) and R01 MH081864 (to Dr O'Neill and J. Piacentini). The remaining authors declare no conflict of interest.

\section{REFERENCES}

Abramowitz J, Franklin M, Street GP, Kozak M, Foa E (2000). Effects of comorbid depression on response to treatment for obsessive-compulsive disorder. Behav Ther 31: 517-528.

Abramowitz JS, Storch EA, Keeley M, Cordell E (2007). Obsessivecompulsive disorder with comorbid major depression: what is the role of cognitive factors? Behav Res Ther 45: 2257-2267.

Aderka IM, Anholt GE, van Balkom AJ, Smit JH, Hermesh H, Hofmann SG et al (2011). Differences between early and late drop-outs from treatment for obsessive-compulsive disorder. $J$ Anxiety Disord 25: 918-923.

Ahmari SE, Dougherty DD (2015). Dissecting Ocd circuits: from animal models to targeted treatments. Depress Anxiety 32: $550-562$.

Alexander GE, DeLong MR, Strick PL (1986). Parallel organization of functionally segregated circuits linking basal ganglia and cortex. Annu Rev Neurosci 9: 357-381.

Baer L, Rauch SL, Ballantine HT Jr., Martuza R, Cosgrove R, Cassem E et al (1995). Cingulotomy for intractable obsessivecompulsive disorder. Prospective long-term follow-up of 18 patients. Arch Gen Psychiatry 52: 384-392.

Bauer DJ, Curran PJ (2005). Probing interactions in fixed and multilevel regression: inferential and graphical techniques. Multivariate Behav Res 40: 373-400.

Beckmann M, Johansen-Berg H, Rushworth MF (2009). Connectivity-based parcellation of human cingulate cortex and its relation to functional specialization. J Neurosci 29: 1175-1190.

Besiroglu L, Uguz F, Saglam M, Agargun MY, Cilli AS (2007). Factors associated with major depressive disorder occurring after the onset of obsessive-compulsive disorder. J Affect Disord 102: 73-79.

Beucke JC, Kaufmann C, Linnman C, Gruetzmann R, Endrass T, Deckersbach T et al (2012). Altered cingulostriatal coupling in obsessive-compulsive disorder. Brain Connect 2: 191-202.

Bijsterbosch J, Smith S, Forster S, John OP, Bishop SJ (2014). Resting state correlates of subdimensions of anxious affect. J Cogn Neurosci 26: 914-926.

Black MD (2005). Therapeutic potential of positive AMPA modulators and their relationship to AMPA receptor subunits. A review of preclinical data. Psychopharmacology (Berl) 179: $154-163$.

Boly M, Sasai S, Gosseries O, Oizumi M, Casali A, Massimini M et al (2015). Stimulus set meaningfulness and neurophysiological differentiation: a functional magnetic resonance imaging study. PLoS One 10: e0125337.

Breiter HC, Rauch SL, Kwong KK, Baker JR, Weisskoff RM, Kennedy DN et al (1996). Functional magnetic resonance imaging of symptom provocation in obsessive-compulsive disorder. Arch Gen Psychiatry 53: 595-606.

Brennan BP, Rauch SL, Jensen JE, Pope HG Jr. (2013). A critical review of magnetic resonance spectroscopy studies of obsessivecompulsive disorder. Biol Psychiatry 73: 24-31.

Brennan BP, Tkachenko O, Schwab ZJ, Juelich RJ, Ryan EM, Athey $\mathrm{AJ}$ et al (2015). An examination of rostral anterior cingulate cortex function and neurochemistry in obsessive-compulsive disorder. Neuropsychopharmacology 40: 1866-1876.

Brown LT, Mikell CB, Youngerman BE, Zhang Y, McKhann GM 2nd, Sheth SA (2016). Dorsal anterior cingulotomy and anterior capsulotomy for severe, refractory obsessive-compulsive disorder: 
a systematic review of observational studies. J Neurosurg 124: 77-89.

Buckner RL, Andrews-Hanna JR, Schacter DL (2008). The brain's default network: anatomy, function, and relevance to disease. Ann NY Acad Sci 1124: 1-38.

Bush G, Luu P, Posner MI (2000). Cognitive and emotional influences in anterior cingulate cortex. Trends Cogn Sci 4: 215-222.

Bush G, Shin LM (2006). The Multi-Source Interference Task: an fMRI task that reliably activates the cingulo-frontal-parietal cognitive/attention network. Nat Protoc 1: 308-313.

Dougherty DD, Baer L, Cosgrove GR, Cassem EH, Price $\mathrm{BH}$, Nierenberg AA et al (2002). Prospective long-term follow-up of 44 patients who received cingulotomy for treatment-refractory obsessive-compulsive disorder. Am J Psychiatry 159: 269-275.

Ernst J, Hock A, Henning A, Seifritz E, Boeker H, Grimm S (2016). Increased pregenual anterior cingulate glucose and lactate concentrations in major depressive disorder. Mol Psychiatry 22: 113-119.

Fitzgerald KD, Stern ER, Angstadt M, Nicholson-Muth KC, Maynor MR, Welsh RC et al (2010). Altered function and connectivity of the medial frontal cortex in pediatric obsessive-compulsive disorder. Biol Psychiatry 68: 1039-1047.

Fox MD, Snyder AZ, Vincent JL, Corbetta M, Van Essen DC, Raichle ME (2005). The human brain is intrinsically organized into dynamic, anticorrelated functional networks. Proc Natl Acad Sci USA 102: 9673-9678.

Franzmeier N, Buerger K, Teipel S, Stern Y, Dichgans M, Ewers M et al (2017). Cognitive reserve moderates the association between functional network anti-correlations and memory in MCI. Neurobiol Aging 50: 152-162.

Glasser MF, Coalson TS, Robinson EC, Hacker CD, Harwell J, Yacoub E et al (2016). A multi-modal parcellation of human cerebral cortex. Nature 536: 171-178.

Habeck C, Steffener J, Rakitin B, Stern Y (2012). Can the defaultmode network be described with one spatial-covariance network? Brain Res 1468: 38-51.

Handwerker DA, Ollinger JM, D'Esposito M (2004). Variation of BOLD hemodynamic responses across subjects and brain regions and their effects on statistical analyses. Neuroimage 21: $1639-1651$.

Hayes A (2013). Introduction to Mediation, Moderation, and Conditional Process Analysis: A Regression-Based Approach. Guilford Press: NY, P85-123 and P207-245.

Johansen-Berg H, Gutman DA, Behrens TE, Matthews PM, Rushworth MF, Katz E et al (2008). Anatomical connectivity of the subgenual cingulate region targeted with deep brain stimulation for treatment-resistant depression. Cereb Cortex 18: 1374-1383.

Keeley ML, Storch EA, Merlo LJ, Geffken GR (2008). Clinical predictors of response to cognitive-behavioral therapy for obsessive-compulsive disorder. Clin Psychol Rev 28: 118-130.

Kennedy SH, Evans KR, Kruger S, Mayberg HS, Meyer JH, McCann $S$ et al (2001). Changes in regional brain glucose metabolism measured with positron emission tomography after paroxetine treatment of major depression. Am J Psychiatry 158: 899-905.

Konova AB, Moeller SJ, Tomasi D, Volkow ND, Goldstein RZ (2013). Effects of methylphenidate on resting-state functional connectivity of the mesocorticolimbic dopamine pathways in cocaine addiction. JAMA Psychiatry 70: 857-868.

Lee HJ, Kwon SM, Kwon JS, Telch MJ (2005). Testing the autogenousreactive model of obsessions. Depress Anxiety 21: 118-129.

Mataix-Cols D, Wooderson S, Lawrence N, Brammer MJ, Speckens A, Phillips ML (2004). Distinct neural correlates of washing, checking, and hoarding symptom dimensions in obsessivecompulsive disorder. Arch Gen Psychiatry 61: 564-576.

Middleton FA (2009). The contribution of anterior cingulate-basal ganglia circuitry to complex behavior and psychiatric disorders. In: Vogt BA (ed). Cingulate Neurobiology and Disease. Oxford University Press: New York, pp 619-632.
Moskal JR, Burgdorf JS, Stanton PK, Kroes RA, Disterhoft JF, Burch RM et al (2016). The Development of Rapastinel (Formerly GLYX-13); a rapid acting and long lasting antidepressant. Curr Neuropharmacol 15: 47-56.

Murphy DL, Moya PR, Fox MA, Rubenstein LM, Wendland JR, Timpano KR (2013). Anxiety and affective disorder comorbidity related to serotonin and other neurotransmitter systems: obsessive-compulsive disorder as an example of overlapping clinical and genetic heterogeneity. Philos Trans R Soc Lond B Biol Sci 368: 20120435.

O'Neill J, Lai TM, Sheen C, Salgari GC, Ly R, Armstrong C et al (2016). Cingulate and thalamic metabolites in obsessivecompulsive disorder. Psychiatry Res 254: 34-40.

Pittenger C, Bloch MH, Williams K (2011). Glutamate abnormalities in obsessive compulsive disorder: neurobiology, pathophysiology, and treatment. Pharmacol Ther 132: 314-332.

Pizzagalli DA (2011). Frontocingulate dysfunction in depression: toward biomarkers of treatment response. Neuropsychopharmacology 36: 183-206.

Power JD, Cohen AL, Nelson SM, Wig GS, Barnes KA, Church JA et al (2011). Functional network organization of the human brain. Neuron 72: 665-678.

Rodriguez CI, Zwerling J, Kalanthroff E, Shen H, Filippou M, Jo B et al (2016). Effect of a novel NMDA receptor modulator, Rapastinel (formerly GLYX-13), in OCD: proof of concept. Am J Psychiatry 173: 1239-1241.

Rosenberg DR, Keshavan MS (1998). A.E. Bennett Research Award. Toward a neurodevelopmental model of of obsessive-compulsive disorder. Biol Psychiatry 43: 623-640.

Ruscio AM, Stein DJ, Chiu WT, Kessler RC (2010). The epidemiology of obsessive-compulsive disorder in the National Comorbidity Survey Replication. Mol Psychiatry 15: 53-63.

Sanacora G, Treccani G, Popoli M (2012). Towards a glutamate hypothesis of depression: an emerging frontier of neuropsychopharmacology for mood disorders. Neuropharmacology 62: 63-77.

Saxena S, Rauch SL (2000). Functional neuroimaging and the neuroanatomy of obsessive-compulsive disorder. Psychiatr Clin North Am 23: 563-586.

Simpson HB, Shungu DC, Bender J Jr., Mao X, Xu X, Slifstein M et al (2012). Investigation of cortical glutamate-glutamine and gamma-aminobutyric acid in obsessive-compulsive disorder by proton magnetic resonance spectroscopy. Neuropsychopharmacology 37: 2684-2692.

Stengler-Wenzke K, Kroll M, Riedel-Heller S, Matschinger $\mathrm{H}$, Angermeyer MC (2007). Quality of life in obsessive-compulsive disorder: the different impact of obsessions and compulsions. Psychopathology 40: 282-289.

Tadayonnejad R, Ajilore O (2014). Brain network dysfunction in late-life depression: a literature review. J Geriatr Psychiatry Neurol 27: 5-12.

Tadayonnejad R, Ajilore O, Mickey BJ, Crane NA, Hsu DT, Kumar A et al (2016a). Pharmacological modulation of pulvinar restingstate regional oscillations and network dynamics in major depression. Psychiatry Res 252: 10-18.

Tadayonnejad R, Klumpp H, Ajilore O, Leow A, Phan KL (2016b). Aberrant pulvinar effective connectivity in generalized social anxiety disorder. Medicine (Baltimore) 95: e5358.

Tukel R, Meteris H, Koyuncu A, Tecer A, Yazici O (2006). The clinical impact of mood disorder comorbidity on obsessive-compulsive disorder. Eur Arch Psychiatry Clin Neurosci 256: 240-245.

Vogt BA, Hof PR, Zilles K, Vogt LJ, Herold C, Palomero-Gallagher $N$ (2013). Cingulate area 32 homologies in mouse, rat, macaque and human: cytoarchitecture and receptor architecture. J Comp Neurol 521: 4189-4204.

Wu GR, Liao W, Stramaglia S, Ding JR, Chen H, Marinazzo D (2013). A blind deconvolution approach to recover effective connectivity brain networks from resting state fMRI data. Med Image Anal 17: 365-374. 
Yeo BT, Krienen FM, Sepulcre J, Sabuncu MR, Lashkari D, Hollinshead $M$ et al (2011). The organization of the human cerebral cortex estimated by intrinsic functional connectivity. $J$ Neurophysiol 106: 1125-1165.

Yucel M, Harrison BJ, Wood SJ, Fornito A, Wellard RM, Pujol J et al (2007). Functional and biochemical alterations of the medial frontal cortex in obsessive-compulsive disorder. Arch Gen Psychiatry 64: 946-955.

Yucel M, Wood SJ, Wellard RM, Harrison BJ, Fornito A, Pujol J et al (2008). Anterior cingulate glutamate-glutamine levels predict symptom severity in women with obsessive-compulsive disorder. Aust N Z J Psychiatry 42: 467-477.

Supplementary Information accompanies the paper on the Neuropsychopharmacology website (http://www.nature.com/npp) 